\title{
Patterns of Atrial Fibrillation After Coronary Artery Bypass Surgery
}

\author{
Jacqueline E. Tamis-Holland, M.D., Marcin Kowalski, M.D., Velisar Rill, M.D., \\ Kamran Firoozi, M.D., and Jonathan S. Steinberg, M.D.
}

From the St. Luke's-Roosevelt Hospital Center and Columbia University, College of Physicians and Surgeons, New York, NY

\begin{abstract}
Background: Atrial fibrillation (AF) is the most common arrhythmia occurring in patients after coronary artery bypass surgery (CABG). The purpose of this study was to determine whether AF characteristics were independently associated with postoperative length of stay (LOS).

Methods: Two hundred ninety consecutive post-CABG patients were examined through a detailed chart review. Baseline, intraoperative, and postoperative variables and the characteristics of AF were recorded. AF episodes were divided into single episodes lasting less than 24 hours (short-lived $\mathrm{AF}$ ) and recurrent or prolonged $\geq 24$ hours of $\mathrm{AF}$ (recurrent/prolonged AF).

Results: AF occurred in 94 (32.4\%) patients. Twenty-six (27.7\%) of AF patients had short-lived $\mathrm{AF}$, and $68(72.3 \%)$ of $\mathrm{AF}$ patients had recurrent/prolonged AF. Patients with recurrent/prolonged AF were older $(P<0.001)$ and more likely to have a history of prior $A F(P<0.001)$ relative to the other groups. Short-lived AF did not prolong LOS $(7.2 \pm 2.1$ days) relative to patients without AF (7.5 \pm 3.9 days), whereas recurrent/prolonged AF significantly prolonged LOS (10.4 \pm 6.1 days, $\mathrm{P}<$ $0.001)$. Multivariate analysis identified postoperative complications, recurrent/prolonged AF, age, and digoxin use as independent predictors of LOS. Recurrent/prolonged AF contributed an additional 1.1 days to LOS after adjusting for baseline clinical differences.

Conclusions: There are heterogenous patterns of AF after cardiac surgery. A substantial minority of AF is short-lived and isolated with no impact on LOS; however, recurrent or prolonged $\mathrm{AF}$ significantly affects LOS.

A.N.E. 2006;11(2):139-144
\end{abstract}

atrial fibrillation; cardiac surgery; coronary artery bypass surgery; length of stay

Atrial fibrillation $(\mathrm{AF})$ is the most common sustained arrhythmia following cardiac surgery, and represents a major cause of morbidity and increased utilization of health care resources. ${ }^{1}$ Although there is an abundance of literature on the prevalence, risk factors, and management of $\mathrm{AF}$ after cardiac surgery, there is scarce data regarding the patterns of postoperative AF. Previous studies have typically grouped all presentations of $\mathrm{AF}$ into a single category regardless of duration, recurrences, hemodynamics, and need for cardioversion. ${ }^{1-6}$ Some studies required postoperative $\mathrm{AF}$ to last as little as 30 seconds to qualify as an endpoint ${ }^{7}$ while other studies required at least 30 minutes of AF. ${ }^{8}$ Although postoperative AF can be short-lived and without sequelae, a number of patients experience recurrences or remain in $\mathrm{AF}$ at the time of discharge from the hospital despite aggressive attempts at treating this arrhythmia. The approach to a patient with a single, asymptomatic, self-limited episode of AF might differ considerably from that of a patient who has recurrent or persistent AF.

We hypothesized that all postoperative AF is not alike. The objective of this study was to describe the patterns of postoperative $\mathrm{AF}$, and to determine which group of patients may warrant early or more aggressive treatment with antiarrhythmic agents or anticoagulation.

\section{METHODS}

\section{Case Selection}

We performed a retrospective analysis through a detailed medical record chart review on 290 
consecutive patients who underwent coronary artery bypass surgery (CABG) at our institution over a 14-month period. Patients were identified through a department log of cardiac surgery cases during this time period. Patients who had concomitant valve replacement were also included in the analysis. All patients underwent conventional "onpump" CABG. Patients who were in normal sinus rhythm (NSR) at the time of the procedure were included in this database. Charts with incomplete or missing recordings were excluded. The primary focus of this study was the development of postoperative $\mathrm{AF}$, with particular interest on the patterns of individual AF episodes.

Cardiac surgery patients were routinely monitored by telemetry for at least 2 or 3 days after surgery, including 1 or 2 days in the intensive care unit, and 1 or 2 more days in the step-down unit. After this, telemetry was, discontinued at the discretion of the attending cardiologist. Telemetry was reinstituted for each episode of AF. In most cases, initial episodes of AF were identified by telemetry monitoring and a hard copy of the rhythm strip and/or the 12-lead ECG was placed in the patient's chart. After reviewing the ECG or telemetry strip, physicians and nurses would then document the arrhythmia in the progress or nursing notes. Every hour telemetry strips were reviewed and the rhythm recorded in the patient's bedside. There was no formal protocol for prophylactic use of $\beta$ adrenergic blocking agents or antiarrhythmic drugs for AF prevention, and therapy was given at the discretion of the attending cardiologist.

\section{Data Collection}

Baseline clinical variables, intraoperative data, and postoperative course including the development of postoperative AF or any serious postoperative event were recorded after careful chart review. Postoperative data collection focused on the occurrence and timing of $\mathrm{AF}$, the duration of the initial episode, as well as the number and duration of subsequent episodes. Our reviewers examined all 12-lead ECGs, telemetry strips, bedside recordings, nursing and progress notes from the patient's chart to identify, and verify each AF episode. We defined postoperative $\mathrm{AF}$ as any episode lasting over 15 minutes. After recording AF episodes in individual patients, we then proceeded to divide patients with AF into two groups. The first group included patients with a single episode of AF lasting
$<24$ hours (Short-lived AF). The second group included patients with prolonged $(\geq 24$ hours) or recurrent $\mathrm{AF}$ (prolonged/recurrent $\mathrm{AF}$ ).

\section{Statistical Analysis}

Continuous variables were expressed as mean \pm SD and analyzed using unpaired 2-tailed Student's $t$-test. Categorical variables were summarized as percentage and compared by chi-square analysis. The 95\% CI was reported where appropriate. Univariate analysis was performed on all variables, and those with a $P$ value of less then 0.1 were included in the multivariate logistic regression analysis. Variables were selected in a step-wise forward selection manner with entry and retention chosen at $\mathrm{P}<$ 0.05 . SPSS Version 10.0 computer software was used for statistical analysis.

\section{RESULTS}

\section{Patterns of AF}

Among 290 patients who underwent cardiac surgery, 94 (32.4\%) developed AF. AF occurred $2.3 \pm 1.2$ days after surgery, with $64 \%$ of AF occurring by the second postoperative day, and $89 \%$ occurring by postoperative day 3 . There was tremendous variation in the duration of the initial $\mathrm{AF}$ episode $(26.1 \pm 51$ hours, range 15 minutes to 288 hours). The median duration of postoperative AF was 7 hours; $69(73 \%)$ patients had an initial episode lasting less then 24 hours and $25(27 \%)$ greater then 24 hours.

Forty-two $(45 \%)$ patients experienced a single AF episode and $26(28 \%)$ experienced a single episode lasting less then 24 hours (short-lived AF). There were $68(72 \%)$ patients who had either recurrent episodes of AF ( $\mathrm{n}=52 ; 55 \%)$ and/or episodes lasting $\geq 24$ hours $(\mathrm{n}=26 ; 28 \%$ ) (recurrent/prolonged $\mathrm{AF}$ ). The average duration of the initial AF episode for patients with short-lived AF and recurrent/prolonged $\mathrm{AF}$ was $4.3 \pm 3.0$ hours and $34.4 \pm 58.3$ hours, respectively $(\mathrm{P}=0.01)$. The average number of recurrences was $3.2 \pm 2.4$ episodes. Nine $(10 \%)$ patients were in $\mathrm{AF}$ at the time of discharge from the hospital.

\section{Baseline, Intraoperative and Postoperative Variables}

Table 1 depicts the baseline variables, and intraoperative and postoperative course for the group 
A.N.E. • April 2006 • Vol. 11, No. 2 • Tamis-Holland, et al. • Patterns of Post-CABG AF • 141

Table 1. Baseline, Intraoperative, and Postoperative Variables

\begin{tabular}{lccc}
\hline Demographics & AF $(\mathbf{n}=\mathbf{9 4})$ & No AF $(\mathbf{n}=\mathbf{1 9 6})$ & P Value \\
\hline Age (years) & $67.0 \pm 9.2$ & $61.7 \pm 9.8$ & $<0.001$ \\
Male gender & $66(70.2 \%)$ & $129(65.8 \%)$ & NS \\
Smoking & $62(66.0 \%)$ & $113(57.7 \%)$ & NS \\
Hypertension & $65(69.1 \%)$ & $140(71.4 \%)$ & NS \\
Diabetes & $34(36.2 \%)$ & $81(41.3 \%)$ & NS \\
Chronic lung disease & $13(13.8 \%)$ & $23(11.7 \%)$ & NS \\
ESRD & $6(6.4 \%)$ & $4(2.0 \%)$ & NS \\
Prior stroke & $7(7.4 \%)$ & $14(7.1 \%)$ & NS \\
Prior MI & $38(40.4 \%)$ & $71(36.2 \%)$ & NS \\
Prior CABG & $8(8.5 \%)$ & $14(7.1 \%)$ & $<0.001$ \\
Prior AF/AFI & $17(18.1 \%)$ & $3(1.5 \%)$ & 0.052 \\
LVEF (\%) & $42.6 \pm 13.0$ & $45.8 \pm 13.0$ & NS \\
Preoperative $\beta$-blocker & $55(58.5 \%)$ & $116(59.2 \%)$ & 0.029 \\
Preoperative digoxin & $13(13.8 \%)$ & $12(6.1 \%)$ & NS \\
Number of grafts & $3.22 \pm 1.02$ & $3.38 \pm 1.83$ & NS \\
Valve surgery & $3(3.2 \%)$ & $1(0.5 \%)$ & NS \\
Postoperative complication & $11(11.7 \%)$ & $32(16.3 \%)$ & \\
\hline
\end{tabular}

$\mathrm{ESRD}=$ end staged renal disease; $\mathrm{MI}=$ myocardial infarction; $\mathrm{CABG}=$ coronary artery bypass graft surgery; $\mathrm{AF}=$ atrial fibrillation; Afl = atrial flutter; $\mathrm{LVEF}=$ left ventricular ejection fraction.

of patients with $\mathrm{AF}$ and for those who remained in NSR throughout the postoperative period, and Table 2 depicts these variables for patients with no AF, short-lived AF, and recurrent/prolonged AF. Patients who developed AF were older $(67.0 \pm 9.2$ years vs $61.7 \pm 9.8$ years, $\mathrm{P}<0.001)$ and were significantly more likely to have a prior history of AF $(18.1 \%$ vs $1.5 \%, \mathrm{P}<0.001)$. Digoxin use prior to surgery was also significantly higher in the patients who developed postoperative AF compared with

Table 2. Baseline, Intraoperative, and Postoperative Variables for the Individual Types of AF

\begin{tabular}{|c|c|c|c|c|c|c|}
\hline Characteristics & $\begin{array}{c}\text { No AF } \\
(n=196)\end{array}$ & $\begin{array}{l}\text { Short-Lived AF } \\
\quad(n=26)\end{array}$ & $\begin{array}{l}\text { Recurrent/ } \\
\text { Prolonged AF } \\
(n=68)\end{array}$ & P Value & $\begin{array}{l}\text { P Value No } \\
\text { AF vs Short- } \\
\text { Lived AF }\end{array}$ & $\begin{array}{l}\text { P Value No AF } \\
\text { vs Recurrent/ } \\
\text { Prolonged AF }\end{array}$ \\
\hline Age (years) & $61.7 \pm 9.8$ & $65.3 \pm 9.5$ & $67.6 \pm 9.1$ & $<0.001$ & 0.08 & $<0.001$ \\
\hline Male gender & $129(65.8 \%)$ & $16(61.5 \%)$ & $50(7 \overline{3} .5 \%)$ & NS & NS & NS \\
\hline Smoking & $113(57.7 \%)$ & $16(61.5 \%)$ & $46(67.6 \%)$ & NS & NS & NS \\
\hline Hypertension & $140(71.4 \%)$ & $19(73.1 \%)$ & $46(67.6 \%)$ & NS & NS & NS \\
\hline Diabetes & $81(41.3 \%)$ & $10(38.5 \%)$ & $24(35.3 \%)$ & NS & NS & NS \\
\hline Chronic lung disease & $23(1.7 \%)$ & $5(19.2 \%)$ & $8(11.8 \%)$ & NS & NS & NS \\
\hline ESRD & $4(2.0 \%)$ & $3(11.5 \%)$ & $3(4.4 \%)$ & 0.039 & 0.009 & NS \\
\hline Prior stroke & $14(7.1 \%)$ & $2(7.7 \%)$ & $5(7.4 \%)$ & NS & NS & NS \\
\hline Prior Ml & $71(36.2 \%)$ & $10(38.5 \%)$ & $28(41.2 \%)$ & NS & NS & NS \\
\hline Prior CABG & $14(7.1 \%)$ & $2(7.7 \%)$ & $6(8.8 \%)$ & NS & NS & NS \\
\hline Prior AF/AFI & $3(1.5 \%)$ & 5 (19.2\%) & $12(17.6 \%)$ & $<0.001$ & $<0.001$ & $<0.001$ \\
\hline LVEF (\%) & $45.8 \pm 13.0$ & $38.9 \pm 12.0$ & $44.0 \pm 13.0$ & 0.035 & 0.011 & NS \\
\hline Preoperative & $116(59.2 \%)$ & $17(65.4 \%)$ & $38(55.9 \%)$ & NS & NS & NS \\
\hline Preoperative digoxin & $12(6.1 \%)$ & $2(7.7 \%)$ & $11(16.2 \%)$ & 0.039 & NS & 0.01 \\
\hline Number of grafts & $3.2 \pm 1.0$ & $3.9 \pm 3.1$ & $3.2 \pm 1.0$ & NS & NS & NS \\
\hline Valve surgery & $1(0.5 \%)$ & $0(0 \%)$ & $3(4.4 \%)$ & 0.049 & NS & 0.023 \\
\hline $\begin{array}{l}\text { Postoperative } \\
\text { complication }\end{array}$ & $32(16.3 \%)$ & $2(7.7 \%)$ & $9(13.2 \%)$ & NS & NS & NS \\
\hline
\end{tabular}

$\mathrm{ESRD}=$ end staged renal disease; $\mathrm{MI}=$ myocardial infarction; $\mathrm{CABG}=$ coronary artery bypass graft surgery; $\mathrm{AF}=$ atrial fibrillation; $\mathrm{Afl}=$ atrial flutter; $\mathrm{LVEF}=$ left ventricular ejection fraction. 
the patients who remained in NSR $(13.8 \%$ vs $6.1 \%$, $\mathrm{P}=0.029$ ). Patients with AF had a trend toward a lower left ventricular ejection fraction than patients in NSR $(42.6 \pm 13.0 \%$ vs $45.8 \pm 13.0 \%, \mathrm{P}=0.052)$. There were no significant differences in intraoperative or postoperative variables between the groups, including the type of surgical procedure performed, the number of vessels bypassed, and the occurrence of postoperative complications. There were a total of three perioperative deaths (1\%). Multivariate analysis on the parameters listed in Table 1 demonstrated that advanced age and a prior history of AF were the only independent predictors of postoperative $\mathrm{AF}(\mathrm{P}<0.001)$.

Patients with recurrent/prolonged AF tended to be older than patients with no AF were more likely to have a history of prior AF and to have undergone valve replacement surgery. Patients with short-lived AF had lower ejection fractions, and were more likely to have a history of prior $\mathrm{AF}$, or renal disease when compared to patients with no AF. There were no notable differences in other intraoperative or postoperative variables among the $\mathrm{AF}$ groups, including the number of vessels bypassed, and the occurrence of postoperative complications. Multivariate analysis on the parameters listed in Table 2 demonstrated that age and prior history of $\mathrm{AF}$ were the only independent predictors of recurrent/prolonged AF $(\mathrm{P}<0.001)$.

\section{Therapy Instituted in the Postoperative Period}

The administration of $\beta$-adrenergic blocking agents early after surgery (on or before postoperative day 2) was similar for patients with no AF (44\%), short-lived AF (54\%), and recurrent/pro- longed $\mathrm{AF}(50 \%)$ When $\mathrm{AF}$ developed, $\beta$-adrenergic blocking agents were used less often for rate control in the group of patients with short-lived AF than patients with recurrent/prolonged AF (Table 3). During the first 24 hours of $\mathrm{AF}$, there was a trend toward less frequent use of antiarrhythmic medications in the group of patients with short-lived $\mathrm{AF}$, $(4 \%$ vs $15 \%, P=0.14)$. Over the entire hospital stay, patients with short-lived AF were also less likely to be treated with IV heparin and antiarrhythmic therapy and were less frequently discharged on warfarin therapy (Table 3). Cardioversion was infrequently employed, but equally used among the two groups. Among the patients cardioverted, $3(38 \%)$ had recurrent AF following cardioversion.

\section{Length of Stay}

The postoperative length of stay (LOS) was significantly longer for patients with AF than patients in NSR $(9.5 \pm 5.5$ days vs $7.5 \pm 3.9$ days, $\mathrm{P}<0.0001)$ However, patients with short-lived AF had similar LOS to patients without AF $(7.2 \pm 2.1$ days vs $7.5 \pm$ 3.9 days, $\mathrm{P}=0.6$ ), while recurrent/prolonged $\mathrm{AF}$ significantly prolonged LOS when compared with those patients with no AF $(10.4 \pm 6.1$ days vs $7.5 \pm$ 3.9 days, $\mathrm{P}<0.0001$ ) (Fig. 1). Multivariate analysis identified postoperative complications, recurrent/prolonged $\mathrm{AF}$, age, and digoxin as independent predictors of LOS (Table 4). After adjusting for other confounding variables, recurrent/prolonged $\mathrm{AF}$ contributed an additional $1.1 \pm 0.3$ days to LOS.

\section{DISCUSSION}

To our knowledge, this is the first report to differentiate $\mathrm{AF}$ as short-lived or recurrent/prolonged,

Table 3. Therapy Instituted in the Postoperative Period

\begin{tabular}{lccc}
\hline & $\begin{array}{c}\text { Short-lived AF } \\
(\mathbf{n}=\mathbf{2 6})\end{array}$ & $\begin{array}{c}\text { Recurrent/Prolonged AF } \\
(\mathbf{n = 6 8 )}\end{array}$ & P Value \\
\hline Early BB therapy (on or before POD 2) & $53.8 \%$ & $50.0 \%$ & NS \\
BB therapy to treat initial AF & $6(23 \%)$ & $33(48 \%)$ & 0.02 \\
CCB therapy to treat initial AF & $5(19 \%)$ & $21(31 \%)$ & NS \\
Digoxin therapy to treat initial AF & $14(54 \%)$ & $42(62 \%)$ & NS \\
Total use of Class I/III antiarrhythmic agents & $1(3.8 \%)$ & $17(25.0 \%)$ & 0.020 \\
Cardioversion & $2(8.0 \%)$ & $6(9.0 \%)$ & NS \\
Heparin & $7(26.9 \%)$ & $50(73.5 \%)$ & $<0.001$ \\
Discharge on warfarin & $3(11.5 \%)$ & $25(36.8 \%)$ & 0.017 \\
\hline
\end{tabular}

$\mathrm{AF}=$ atrial fibrillation; $\mathrm{BB}=\beta$-adrenergic blocking agents; $\mathrm{CCB}=$ calcium channel blockers; $\mathrm{POD}=$ postoperative day. 


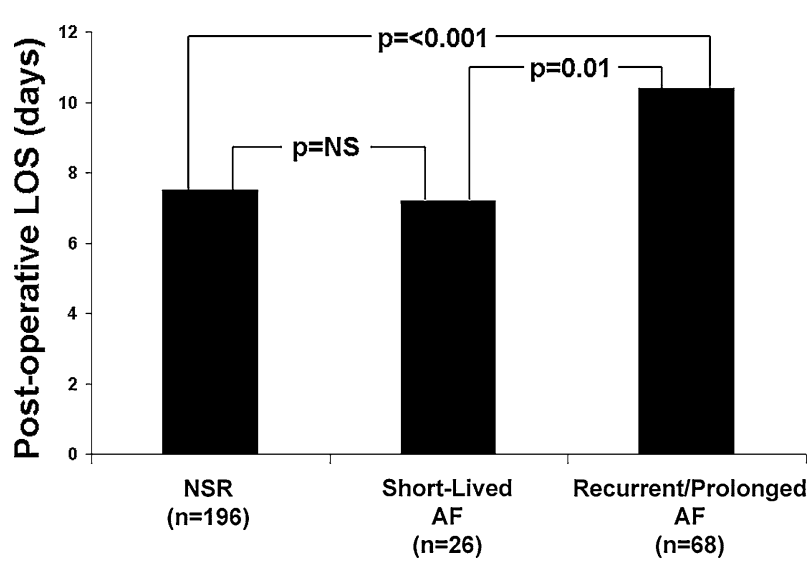

Figure 1. A comparison of postoperative LOS for patients with normal sinus rhythm (NSR), short-lived AF and recurrent/prolonged AF.

and demonstrate that such differences in $\mathrm{AF}$ patterns impact the LOS after CABG. Not surprisingly, we found a heterogeneous pattern of $\mathrm{AF}$, consistent with the hypothesis that postoperative AF is not a uniform clinical condition. This information is important to physicians caring for cardiac surgery patients, as it helps to identify who might require early and aggressive therapy for postoperative AF.

In the current study, AF was common after cardiac surgery, occurring in about one third of all patients. The vast majority of patients developed $\mathrm{AF}$ by the third postoperative day. Multivariate analysis demonstrated that postoperative AF was significantly more frequent in older patients, or patients with a prior history of AF. These findings are consistent with reports from previous database analyses. ${ }^{1-3,5,9}$

There was a large variance in the duration of the initial AF episode, with one fourth of patients experiencing AF for over 24 hours. Approximately

Table 4. Independent Predictors of Prolonged Length of Stay

\begin{tabular}{lccc}
\hline Variable & $\begin{array}{c}\text { Regression } \\
\text { Coefficient }\end{array}$ & $\begin{array}{c}\text { Standard } \\
\text { of Error }\end{array}$ & P-Value \\
\hline Complications & 3.56 & 0.68 & $<0.001$ \\
Recurrent/prolonged & 1.14 & 0.30 & $<0.001$ \\
$\quad$ AF & & & \\
$\begin{array}{l}\text { Digoxin } \\
\text { Age (for every }\end{array}$ & 3.20 & 0.89 & $<0.001$ \\
$\quad$ 10 years) & 0.74 & 0.25 & 0.003 \\
\hline
\end{tabular}

$\mathrm{AF}=$ atrial fibrillation half of all patients with postoperative AF experienced subsequent AF episodes, while three fourths of all patients with $\mathrm{AF}$ had either prolonged or recurrent AF (recurrent/prolonged AF). Patients with recurrent/prolonged $\mathrm{AF}$ were as likely to be treated routinely with $\beta$-adrenergic blocking agents in the early postoperative period as patients without $\mathrm{AF}$, or patients with short-lived AF. Recurrent/prolonged $\mathrm{AF}$ was not associated with a higher complication rate, but was associated with more frequent use of IV anticoagulation, antiarrhythmic therapy, and discharge on warfarin therapy.

LOS was significantly higher for patients with AF when compared with patients in NSR. However, when grouped according to the pattern of AF, LOS was only prolonged for patients with recurrent/prolonged AF. Multivariate analysis demonstrated that recurrent/prolonged AF was an independent predictor of prolonged LOS, and contributed an additional 1.1 days to hospital LOS after accounting for other confounding variables. In a recent study, examining risk factors and outcome of $\mathrm{AF}$ after cardiac surgery, ${ }^{10}$ the authors reported a $42 \%$ incidence of recurrent $\mathrm{AF}$ among patients with postoperative AF. They too demonstrated that patients with recurrent AF had a longer LOS compared with patients with a single episode of AF. However, the longer LOS for patients with recurrent AF appeared to be related to a higher rate of postoperative complications. In our study, patients with recurrent/prolonged AF had similar complication rates, but were more likely to be treated with anticoagulation, and antiarrhythmic therapy. We attribute the increased LOS for patients with recurrent/prolonged AF to be a result of the additional days used to observe these patients and/or to initiate and titrate therapy.

In view of this information, it may be worthwhile to consider a strategy that focuses on rate control and the initiation of early anticoagulation once recurrent/prolonged AF is recognized. This would incorporate the routine use of $\beta$-adrenergic blocking agents for all postoperative patients (unless there is a contraindication), and possibly the institution of low molecular weight heparin as a bridge to warfarin therapy once recurrent/prolonged AF is recognized. ${ }^{11}$ Whether restoration of sinus rhythm should be attempted is still a matter of debate. Recent data ${ }^{12}$ suggest little difference between a primary rate control strategy and a strategy that restores sinus rhythm (antiarrhythmic therapy with or without electrical cardioversion). Since most AF 
resolves by 8 weeks after surgery, ${ }^{12}$ anticoagulation can subsequently be discontinued during followup. For the small number of patients who remain in $\mathrm{AF}$ beyond 8 weeks, cardioversion may then be offered. Further investigation is needed to determine whether this approach improves LOS, while maintaining optimal patient care.

\section{Study Limitations}

This report is based on a retrospective, though detailed, data collection and review, which focused on the occurrence and characteristics of postoperative AF. It is possible that short, asymptomatic arrhythmic episodes were missed after discontinuation of telemetry, hence potentially overestimating the number of patients with "short-lived and nonrecurrent AF." The only true way to identify all AF episodes would be to perform a prospective registry with continuous telemetry until discharge from the hospital. There was no uniform protocol for AF management: rate and rhythm control, as well as the use of anticoagulation were given at the discretion of the attending physician, potentially biasing the results. However, most cardiologists and surgeons at our institution have similar practice patterns based on hospital and expert opinion recommendations. Finally, long-term follow-up of postdischarge AF outcome was not performed.

\section{CONCLUSIONS}

AF is common after cardiac surgery, and has a heterogeneous pattern. A substantial minority of $\mathrm{AF}$ is short-lived and isolated with no impact on LOS; however, recurrent or prolonged AF significantly prolongs LOS. Efforts should focus on aggressively treating patients who develop the features of postoperative recurrent/prolonged AF in an attempt to improve LOS and decrease resource utilization.

\section{REFERENCES}

1. Aranki SF, Shaw DP, Adams DH, et al. Predictors of atrial fibrillation after coronary artery surgery. Current trends and impact on hospital resources. Circulation 1996;94(3):390-397.

2. Leitch JW, Thomson D, Baird DK, et al. The importance of age as a predictor of atrial fibrillation and flutter after coronary artery bypass grafting. J Thorac Cardiovasc Surg 1990;100(3):338-342.

3. Fuller JA, Adams GG, Buxton B. Atrial fibrillation after coronary artery bypass grafting. Is it a disorder of the elderly? J Thorac Cardiovasc Surg 1989;97(6):821-825.

4. Creswell LL, Schuessler RB, Rosenbloom M, et al. Hazards of postoperative atrial arrhythmias. Ann Thorac Surg 1993;56(3):539-549.

5. Mathew JP, Parks R, Savino JS, et al. For the MultiCenter Study of Perioperative Ischemia Research Group. Atrial fibrillation following coronary artery bypass graft surgery: Predictors, outcomes, and resource utilization. J Am Med Assoc 1996;276(4):300-306.

6. Almassi GH, Schowalter T, Nicolosi AC, et al. Atrial fibrillation after cardiac surgery: A major morbid event? Ann Surg 1997;226(4):501-511.

7. Rubin DA, Nieminski KE, Reed GE, et al. Predictors, prevention, and long-term prognosis of atrial fibrillation after coronary artery bypass graft operations. J Thorac Cardiovasc Surg 1987;94(3):331-335.

8. Tamis JE, Steinberg JS. Atrial fibrillation independently prolongs hospital stay after coronary artery bypass surgery. Clin Cardiol 2000;23(3):155-159.

9. Frost L, Molgaard H, Christiansen EH, et al. Low vagal tone and supraventricular ectopic activity predict atrial fibrillation and flutter after coronary artery bypass grafting. Eur Heart J 1995;16(6):825-831.

10. Joseph PM, Manuel LF, Iulia CT, et al. A multicenter risk index for atrial fibrillation after cardiac surgery. J Am Med Assoc 2004;291:1720-1729.

11. Steinberg JS. Postoperative atrial fibrillation: a billion dollar problem. J Am Coll Cardiol 2004;43:1001-1003.

12. Lee JK, Klein GJ, Krahn AD, et al. Rate-control versus conversion strategy in postoperative atrial fibrillation: A prospective, randomized pilot study. Am Heart J 2000;140(6):871-877. 